\title{
NOTE ON A PAPER BY MANDELBROJT AND MACLANE
}

\author{
BY \\ JACQUELINE FERRAND
}

The results obtained by J. Dufresnoy and J. Ferrand( $\left.{ }^{1}\right)$ enable us to extend Theorems I, II, and III of the preceding paper to more general strip regions.

Let $g_{i}(\sigma)>0(i=1,2)$ be defined and continuous for $\sigma \geqq a(-\infty \leqq a<\infty)$ with $\lim _{\sigma \rightarrow \infty} g_{i}(\sigma)=\pi / 2$. Let

$$
S(\sigma)=\pi \int_{c}^{\sigma} \frac{d u}{g_{1}(u)+g_{2}(u)} .
$$

Let $\Delta_{\mathrm{g}}$ be the domain in the $s$-plane $(s=\sigma+i t)$ defined by $-g_{1}(\sigma)<t<g_{2}(\sigma)$. If $g_{1}(\sigma)=g_{2}(\sigma)$ we have the symmetrical domain considered above.

Lemmas I, II, III, and IV are true for the new domain if we suppose that $g_{1}(\sigma)$ and $g_{2}(\sigma)$ separately satisfy all the conditions given for $g(\sigma): g_{1}(\sigma)$ and $g_{2}(\sigma)$ must be of bounded variation( $\left.{ }^{2}\right)$ and satisfy the condition (11), $\left|g_{i}^{\prime}(\sigma)\right|<A$, $g_{i}^{\prime}(\sigma+h)-g_{i}^{\prime}(\sigma)>-A h$. The proofs of Theorems I, II, and III are the same, with the new function $S(\sigma)$.

The University of Caen, Caen, France

Received by the editors August 18, 1946.

(1) C. R. Acad. Sci. Paris vol. 220 (1945) pp. 189, 873.

(2) In fact the conclusions hold if this first condition is replaced by $\int^{\infty}\left|g_{i}^{\prime}(\sigma)\right|^{2} d \sigma<\infty$. 